\title{
Research on Estimation of Wheat Chlorophyll Using Image Processing Technology
}

\author{
Liya Zhou ${ }^{1,}$, , Yingchun Yuan ${ }^{1}$, Yufei Song ${ }^{1}$ and Bin Wang ${ }^{1}$ \\ ${ }^{1}$ Hebei Agricultural University, School of Information Science and Technology, 071001, China
}

\begin{abstract}
In the field environment, the chlorophyll values of wheat leaves are estimated based on image processing. The estimation model of wheat color features and SPAD(chlorophyll value) is established. The model is tested by applying them into five different nitrogen fields, and the experimental results show that the actual value and the estimated value of the model are correlated with high significance.
\end{abstract}

\section{Introduction}

As a big agricultural country, China has a lot of different kinds of crops. Wheat is one of the most important economic crops so that the researching on its nutritional status is of great significance ${ }^{[1]}$. At different nitrogen levels, the color characteristics of wheat leaves are different, so the nitrogen nutrition characteristics of the crop could be judged by leaf color, and the fertilization was instructed to alleviate the waste of fertilizer and the phenomenon of land plate ${ }^{[2]}$. For a long time, the wheat experts and growers often evaluate the depth of leaf color on their naked eyes ${ }^{[3]}$. This method is time consuming and laborious. The evaluation results are often qualitative and no quantitative indicators, and they are also affected by the experience of the observers. In contrast, computer recognition is more accurate than the human eyes ${ }^{[4]}$. Guo Wenchuan et.al [5] extracted the corn leaf color characteristics, the green standard value $G /(R+G+B)$ was chosen to characterize the nitrogen content of maize leaves. Zhang yan'e et.al ${ }^{[6]}$ used the digital image technique to obtain the color characteristics (R, G, B, H, I, $\mathrm{S})$ of the cucumber leaf image under natural light. The results show that the color components $\mathrm{G}$ and $\mathrm{H}$ are related to the nitrogen content under the same light, and the method can be used to predict the nitrogen content of cucumber, but the model is affected by the light intensity. Zhu Hong et.al ${ }^{[7]}$ extracted the color characteristics of the wheat canopy image, and evaluated the nutritional status of the wheat on the low, medium and high nitrogen levels by the histogram calculation of the RGB and HIS systems. In actually, wheat's growth is affected by the natural environment, so the research results are only a trend, which cannot accurately evaluate wheat nitrogen nutrition. Shi lei et. al ${ }^{[8]}$ established an estimation model between wheat color characteristics and chlorophyll. The results show that the chlorophyll status of wheat populations could be estimated effectively by wheat color characteristics. The disadvantage of this estimation model is that the extraction of color features is not as accurate as it should be owing to the growth period and the light effects.

It is found that the jointing period of wheat is the most obvious period of the relationship between color characteristics of wheat and SPAD [9]. Therefore, in the jointing period of wheat, using the image processing technology to extract the color characteristics of wheat leaf in the field environment, the estimation model between color and SPAD was established.

\section{Experimental data collection}

The experiment was carried out in the Huangtuo Village (Qingyuan County, Baoding city) between the years of 2016 and 2017. In the field environment, selecting Ji Mai 22 as experimental varieties, and selecting 15 test sites numbered block from 1 to 15 . Experimental urea (containing $46 \% \mathrm{~N}$ ) is used as nitrogen fertilizer. In this study, five nitrogen levels were selected, respectively as $\mathrm{N} 255$ (pure $\mathrm{N}, 255 \mathrm{~kg} / \mathrm{hm} 2$ ), N330 (pure N, $330 \mathrm{~kg} /$ hm2), plots and N-level (N), N180 (pure N, $180 \mathrm{~kg} / \mathrm{hm} 2$ ). The corresponding relationship between plots and $\mathrm{N}$ levels is shown in Table 1. 
Table 1. The map of block and the level of nitrogen application

\begin{tabular}{|c|c|c|c|c|c|}
\hline \multirow{2}{*}{ Block number } & \multicolumn{5}{|c|}{ Nitrogen level / (kg/hm2) } \\
\hline & $\mathbf{0}$ & 100 & 180 & 255 & 330 \\
\hline $1,8,10$ & & & & $※$ & \\
\hline $2,9,13$ & & $※$ & & & \\
\hline $3,5,12$ & & & & & $※$ \\
\hline $4,7,14$ & & & $※$ & & \\
\hline $6,11,15$ & $※$ & & & & \\
\hline
\end{tabular}

In the jointing period of wheat, the data were collected on May 6, 2017 which included the acquisition of wheat chlorophyll value and image acquisition of wheat leaf. The leaf image was taken after the SPAD value was measured, that is, the blade image is in one-to-one correspondence with the SPAD value.

The SPAD value of wheat was obtained by chlorophyll meter ${ }^{[10]}$. Fifteen representative wheat canopy leaves were selected from each of the 15 experimental sites, with a total of 450 sample data. Take leaf from the leaf base 1/3 and measure the SPAD value, then repeat the measurement 3 times, eventually, take the average as the leaf chlorophyll content.

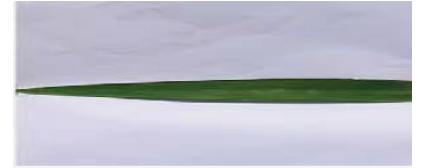

A) The Original Image

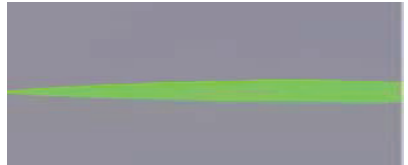

B) The Normalized Image

Figure.1 The picture before and after image processing

The wheat leaf image is captured with the Sony FDR-AXP35 4K HD camcorder and stored in BMP format. Pictures are taken when the weather is clear (to avoid the light being too strong or too dark) and without wind. The photo time is fixed within 12 am given or within 2 hours. When the lens is at the position of $100 \mathrm{~cm}$ perpendicular to wheat leaf, the image can be taken. When taking the pictures, put a piece of pure A4 paper closer to the wheat leaves, in order to get a better extraction of the wheat leaf color. Before taking pictures, fix the camera internal parameters first, and set the exposure speed at about $1 / 650 \mathrm{~s}$, the aperture value about F6.0. Get the picture shown in Figure 1A.

\section{Modeling}

\subsection{Image Preprocessing}

After the wheat leaf image is collected, it is preprocessing first. The order operation includes normalization, color segmentation and morphological processing, in which the particularly important operations are normalization and color segmentation.

\subsubsection{Normalization}

The light effects on the extraction of color features are very significant. Given one piece of wheat leaf, its color information is recorded under different lights, which are shown in Table 2.

Table2. Color characteristic values under different lights.

\begin{tabular}{ccc}
\hline $\mathbf{I}$ & $\mathbf{G}$ & $\mathbf{B}$ \\
\hline 0.3 & 23 & 12 \\
0.55 & 60 & 26 \\
0.70 & 80 & 39 \\
0.85 & 97 & 52 \\
1.0 & 112 & 65 \\
1.15 & 124 & 76 \\
1.3 & 135 & 88 \\
1.45 & 144 & 97 \\
\hline
\end{tabular}

\begin{tabular}{lll}
\hline 1.6 & 151 & 106 \\
\hline
\end{tabular}

In table 2 , the symbol I indicates the ratio between the brightness and darkness of the light, and $R, G, B$ respectively stand for the values in the red channel, the green channel, and the blue channel of a leaf. $I=1$ represents the ideal light.From table 2, the values of $R, G$ and $\mathrm{B}$ are positively correlated with the light. However, the changes of I are not the same as the change of R, G and $\mathrm{B}$. Therefore, the results show that the light has an effect on the color feature extraction.

As the leaf image is carried out in the field environment, the light impact on it cannot be avoided ${ }^{[11]}$. To avoiding the light impact on the color feature extraction, all the images need to be normalized, which can be achieved by using the formula (1) as follow.

$$
\left\{\begin{array}{l}
r=\frac{R}{\sqrt{R^{2}+G^{2}+B^{2}}} \\
g=\frac{G}{\sqrt{R^{2}+G^{2}+B^{2}}} \\
b=\frac{B}{\sqrt{R^{2}+G^{2}+B^{2}}}
\end{array}\right.
$$

Where, the $\mathrm{R}, \mathrm{G}, \mathrm{B}$ respectively represents the color components of the original image, and $\mathrm{r}, \mathrm{g}, \mathrm{b}$ stands for the normalized color components. The normalized image is shown in Figure 1B. 


\subsubsection{Color Segmentation of lages}

Threshold segmentation can be divided into gray scale and color segmentation. Color segmentation is adopted in this paper in order to preserve better color information. Color segmentation can affect the accuracy of color feature extraction. Segmentation technologies have edge segmentation technology, threshold segmentation technology and regional segmentation technology. Among them, the threshold segmentations include global threshold segmentation, Otsu threshold segmentation and iterative threshold segmentation [12]. This study uses iterative threshold segmentation. Its procedure is described as follows:

(1) Set the parameters, find the minimum gray value $\mathrm{Z} 0$ and the maximum gray value $\mathrm{Z1}$ in the image, and select the initial estimation parameter $\mathrm{T} 1=(\mathrm{Z} 0+\mathrm{Z} 1) / 2$.

(2) The image is divided by the threshold value T1. The image is divided into two parts: the gray value is greater than $\mathrm{T} 1$ for the $\mathrm{C} 1$ part, less than the $\mathrm{T} 1$ part of the $\mathrm{C} 2$.

(3) Find the average gray values $\mathrm{u} 1$ and $\mathrm{u} 2$ for the $\mathrm{C} 1$ and $\mathrm{C} 2$ pixels, and the new threshold $\mathrm{T} 2=(\mathrm{u} 1+\mathrm{u} 2) / 2$.
(4) If $|\mathrm{T} 2-\mathrm{T} 1|<\mathrm{T} 0$, then $\mathrm{T} 2$ is the optimal threshold; otherwise, $\mathrm{T} 1=\mathrm{T} 2$, and repeat steps $(2) \sim(4)$ until the optimal threshold is obtained.

(5) The threshold image is multiplied by the RGB image to obtain a split image.

The image after color division is shown in Fig. 1C.

\subsection{Color features selection}

The color features can be described the surface characteristics of the image area, so the color features are widely used in image recognition. The related literatures has shown that SPAD is closely related to the color features. In this paper, 12 characteristic parameters are required to study the relationship between SPAD and color characteristics. They are the first moments(R, G, B), the second moments(SR, SG, SB), the normalized first moments(r, g, b), and the normalized second moments $(\mathrm{Sr}$, $\mathrm{Sg}, \mathrm{Sb})$. Table 3 shows the results of the 12 characteristic parameters extracted from 450 samples (where id shows the leaf number and avg is the mean value).

Table 3. Color characteristic values.

\begin{tabular}{lllllllllllll}
\hline id & R & G & B & SR & SG & SB & r & g & b & Sr & Sg & Sb \\
\hline 1 & 72 & 97 & 57 & 15 & 12 & 22 & 136 & 187 & 104 & 5 & 11 & 24 \\
2 & 69 & 89 & 58 & 15 & 12 & 20 & 138 & 180 & 115 & 5 & 9 & 20 \\
3 & 46 & 65 & 36 & 15 & 13 & 22 & 129 & 191 & 98 & 10 & 13 & 40 \\
4 & 56 & 77 & 45 & 16 & 12 & 27 & 132 & 189 & 100 & 7 & 10 & 38 \\
5 & 39 & 52 & 36 & 15 & 13 & 16 & 130 & 181 & 123 & 8 & 8 & 12 \\
6 & 54 & 81 & 36 & 16 & 15 & 19 & 130 & 199 & 86 & 8 & 9 & 30 \\
7 & 38 & 51 & 30 & 13 & 12 & 15 & 134 & 187 & 106 & 9 & 10 & 24 \\
8 & 47 & 64 & 39 & 15 & 13 & 19 & 133 & 187 & 107 & 7 & 11 & 23 \\
$\ldots$ & $\ldots$ & $\ldots$ & $\ldots$ & $\ldots$ & $\ldots$ & $\ldots$ & $\ldots$ & $\ldots$ & $\ldots$ & $\ldots$ & $\ldots$ & $\ldots$ \\
443 & 65 & 90 & 66 & 20 & 16 & 24 & 126 & 180 & 127 & 9 & 12 & 16 \\
444 & 46 & 58 & 38 & 13 & 12 & 14 & 139 & 179 & 115 & 6 & 11 & 15 \\
445 & 36 & 49 & 32 & 13 & 12 & 14 & 133 & 183 & 115 & 8 & 10 & 16 \\
446 & 42 & 60 & 40 & 16 & 13 & 18 & 126 & 184 & 119 & 10 & 10 & 22 \\
447 & 57 & 80 & 48 & 21 & 18 & 24 & 130 & 190 & 105 & 10 & 14 & 27 \\
448 & 37 & 52 & 32 & 14 & 12 & 15 & 131 & 188 & 110 & 8 & 11 & 18 \\
449 & 30 & 40 & 30 & 13 & 11 & 15 & 127 & 180 & 125 & 8 & 10 & 20 \\
450 & 55 & 77 & 52 & 17 & 13 & 23 & 127 & 185 & 117 & 8 & 11 & 25 \\
avg & 53 & 72 & 39 & 15 & 13 & 19 & 134 & 188 & 100 & 8 & 11 & 26 \\
\hline
\end{tabular}

The first moments $\mathrm{R}, \mathrm{G}, \mathrm{B}$ and normalized $\mathrm{r}, \mathrm{g}, \mathrm{b}$ are the components representing the color information, and the second moments SR, SG, SB and the normalized second moments $\mathrm{Sr}, \mathrm{Sg}, \mathrm{Sb}$ indicate whether the distribution of color information is uniform.

From Table 3, the first moment of the R, G, B values become larger after normalization, whereas the second moments of $\mathrm{Sr}$ and $\mathrm{Sg}$ become smaller. The results show that the influence of light reflection and shadow on the image is significant in different images. After the normalization, its color distribution span becomes smaller, and the color information is more stable, more suitable for the color diagnosis.

\subsection{SPAD prediction model}

The correlation between the 12 color characteristics of Table 3 and SPAD was analyzed by SPSS22.0 data analysis software, and the correlation was established as shown in Table 4. 
Table 4. Correlation between color features and SPAD value.

\begin{tabular}{ccc}
\hline Number & Color feature & Correlation coefficient \\
\hline 1 & $\mathrm{R}$ & -0.545 \\
2 & $\mathrm{G}$ & -0.589 \\
3 & $\mathrm{~B}$ & 0.183 \\
4 & $\mathrm{SR}$ & 0.009 \\
5 & $\mathrm{SG}$ & -0.191 \\
6 & $\mathrm{SB}$ & -0.247 \\
7 & $\mathrm{r}$ & -0.704 \\
8 & $\mathrm{~g}$ & -0.619 \\
9 & $\mathrm{~b}$ & 0.794 \\
10 & $\mathrm{Sr}$ & 0.391 \\
11 & $\mathrm{Sg}$ & 0.065 \\
12 & $\mathrm{Sb}$ & -0.441 \\
\hline
\end{tabular}

Table 4 lists the correlation between color characteristics and SPAD, and the higher correlation color features are selected to establish the regression model. Experiments show that the correlation of the color eigenvalues of $\mathrm{r}, \mathrm{g}, \mathrm{b}$ and SPAD is notable. So the regression equation is described as follow (2), and its coefficient $\mathrm{R}$ equals to 0.85 .

$$
S P A D=59.098-0.432 r+0.100 g+0.275 b
$$

\section{Model test}

The independent samples test the above estimation equation between color feature and SPAD value. Three parameters are selected to evaluate it such as the relative error of the estimated results(E), root mean square error (ERMS), and accuracy (R2). The samples are 100. The corresponding test results are shown in Table 5 below.

Table 5. Test results.

\begin{tabular}{cccc}
\hline Number & Relative error & Root mean square error & Accuracy \\
\hline $1 \sim 20$ & $0.043-0.137$ & 1.7263 & 0.8935 \\
\hline $21 \sim 40$ & $0.041-0.135$ & 1.7132 & 0.9100 \\
\hline $41 \sim 60$ & $0.042-0.139$ & 1.6745 & 0.8973 \\
\hline $61 \sim 80$ & $0.044-0.137$ & 1.6851 & 0.8896 \\
\hline $81 \sim 100$ & $0.046-0.137$ & 1.6737 & 0.8965 \\
\hline
\end{tabular}

From table 5, the estimated values of the model are significantly correlated with the measured values. The average of corresponding mean square error is less than 1.8 , the average of relative error is between $4.3 \%$ and $13.7 \%$, the average of accuracy is 0.8974 . So all the results indicates that the estimate model has achieved a high accuracy.

\section{Conclusion}

In this study, image processing technology has been used to establish the estimation model of wheat color characteristics and chlorophyll. First, the color characteristics information of wheat leaf has been extracted by using color segmentation. Then the relationship between the color characteristics of wheat leaf and SPAD are also analyzed. At last, the estimation model is established ant it can achieve higher accuracy by testing in 450 image samples. However, this study is still inadequate; SPAD and partial color characteristics of the low correlation still need further study for the reason that the images are collected in the field environment, and the leaf color information collection is affected by the light impact. Otherwise, the normalized operation can remove some darker colors, so the collected color information is incomplete.

\section{References}

1. Fusuo. Zhang, Kuiqing.Wang, Weifeng. Zhang, etc., Acta Pedologica Sinica, 45(5), 915-92, (2008).

2. Funing Wu. Beijing, China Agricultural University, (2004).

3. Yuanyuan. Shi,Jinsong. Deng, Lisu. Chen, etc., $S p$ ectr-oscopy and Spectral Analysis, 30, 214-219, (2 010).

4. Tao. Liu, Xiaochun. Zhong, Chengming. Sun, etc., Scie-ntia AgriculturaSinica, 47 (4), 664-674, (201 4).

5. Wenchuan. Guo, Chaochao. Zhou, Wenting. Han, Tra-nsactions of the Chinese Society of Agricultura $l$ Machinery, 42 (1),275-280, (2014).

6. Yanye. Zhang, Minzan. Li,Transaction of the Chin -ese Society of Agricultural Machinery, 21, 102-10 5, (2005).

7. Hong. Zhu, Limin. Zheng, Jianling. Yin, Fu'ning. Wu, Transactions of the Chinese Society of Agricu ltural Machinery, 26 (2), 16-20, (2010). 
8. Lei. Shi, Xiaodan. Pang, Cheng. Qian, etc., Journ al of Taiyuan University of Technology, 47(2016).

9. Zhongbo. Xiao, Liangliang. Jia, Xinping. Chen, $C$ hinese Agricultural Science Bulletin,24 (8),448-453, (2008).

10. Tiancheng. Ai, Fangmin. Li, Zhian. Zhou, etc., Jo urnal of Hubei Agricultural College, 20(1), 6-8, (2000).

11. Jiayue. Chen, Xia. Yao, Fen. Huang, etc., Transac tions of the Chinese Society of AgriculturalMachin ery, 32 (4), 163-170, (2016).

12. Zhibin. Zhang, Xiwen. Luo, Ying. Zang, etc., Tra nsa-ctions of the Chinese Society of Agricultural Machinery ,27(7), 183-189, (2011) 